\title{
Far-Ultraviolet Observation of the Globular Cluster NGC 6397
}

\author{
A. Dieball ${ }^{1,2 \star}$, A. Rasekh ${ }^{3}$, C. Knigge ${ }^{4}$, M. Shara ${ }^{5}$, D. Zurek ${ }^{5}$ \\ ${ }^{1}$ Argelander Institut für Astronomie der Universität Bonn, Auf dem Hügel 71, 53121 Bonn, Germany \\ ${ }^{2}$ Helmholtz-Institut für Strahlen und Kernphysik, Nussallee 14-16, 53115 Bonn, Germany \\ ${ }^{3}$ Oskar Klein Centre, Department of Astronomy, Stockholm University,SE-106 91 Stockholm, Sweden \\ ${ }^{4}$ Physics and Astronomy, University of Southampton, Southampton SO17 1BJ, UK \\ ${ }^{5}$ Department of Astrophysics, American Museum of Natural History, New York, NY 10024, USA
}

Accepted XXX. Received YYY; in original form ZZZ

\begin{abstract}
We present an observational far-UV (FUV) and near-UV (NUV) study of the core region of the globular cluster NGC6397. The observations were obtained with the Space Telescope Imaging Spectrograph (STIS, FUV), and the Wide Field Camera 3 (WFC3, NUV) on board the Hubble Space Telescope. Here, we focus on the $U V$ bright stellar populations such as blue stragglers (BSs), white dwarfs (WDs) and cataclysmic variables (CVs). We present the first $F U V-N U V$ color-magnitude diagram (CMD) for this cluster. To support our classification of the stellar populations, we compare our FUV - NUV CMD with optical data from the ACS Survey of Galactic Globular Clusters. The FUV - NUV CMD indicates 16 sources located in the WD area, and ten BSs within the $25^{\prime \prime} \times 25^{\prime \prime}$ of the STIS $F U V$ data. Eighteen Chandra X-ray sources are located within the $F U V$ field of view. Thirteen of those have a $N U V$ counterpart, of which nine sources also have a $F U V$ counterpart. Out of those, five sources are previously suggested CVs, and indeed all five are located in the WD/CV region in our $F U V-N U V$ CMD. Another CV only has a FUV but no NUV counterpart. We also detect a $N U V$ (but no $F U V$ ) counterpart to the MSP located in the core of this cluster. The NUV lightcurves of the CVs and MSP show flickering behaviour typical of CVs. We found that the BSs and CVs are the most centrally concentrated population. This might be an effect of mass segregation or indicate the preferred birth place of BSs and CVs via dynamical interactions in the dense core region of GCs. HB stars are the least centrally concentrated population and absent in the innermost area of the core.
\end{abstract}

Key words: globular clusters: individual (NGC 6397) - ultraviolet: stars - cataclysmic variables - white dwarfs - techniques: photometric

\section{INTRODUCTION}

Globular clusters (GCs) are the oldest and most massive stellar aggregates in our Galaxy. The stellar density in the cores of GCs can go up to $10^{6}$ stars $^{\mathrm{pc}^{-3}}$. Therefore, close encounters or even direct collisions with resulting mergers between the cluster members can be quite frequent, resulting in dynamically formed new, exotic stellar populations such as blue stragglers (BSs), cataclysmic variables (CVs), lowmass X-ray binaries (LMXBs), millisecond pulsars (MSPs) and other close binary (CB) systems. CBs are important for the evolution of GCs, since the binding energy of a few CBs can be comparable to the binding energy of the entire

^ E-mail: adieball@astro.uni-bonn.de
GC. By transferring their orbital energy to passing single stars, CBs can have a significant impact on the dynamical evolution of the cluster (see Elson et al. 1987; Hut et al. 1992, and references therein). Heggie \& Giersz (2009) and Giersz \& Heggie (2009) performed N-body and Monte Carlo simulations of the two closest GCs NGC 6397 and M 4 to study the evolution of their central structure. Both clusters are similar in mass, but show different surface brightness profiles. The simulations suggest that both clusters are corecollapsed, but the different surface brightness profiles can be explained by fluctuations of the core, which are influenced by dynamically active binaries (Heggie \& Giersz 2009). Interacting $\mathrm{CBs}$ like CVs are an important subset of CBs and can be used to trace the overall CB population in a cluster. CVs may form via ordinary evolution of primordial binaries, 
and this formation mechanism can already account for significant numbers of CVs (see, e.g., Davies 1997), but dynamical formation scenarios like tidal capture and three body encounters can also create CVs in the dense cores of GCs. For example, Di Stefano \& Rappaport (1994), Shara \& Hurley (2006), and Ivanova et al. (2006) discussed several primordial and dynamical formation scenarios and predicted a few 100 CVs in a GC like 47 Tuc. Pooley \& Hut (2006) suggested that the CVs in dense GCs are predominantly dynamically formed.

How can we study the CV and other exotic stellar populations in a GC observationally? Most exotica like CVs and LMXBs are optically faint, and the cores of GCs, their birth sites, are crowded, making their detection even more difficult. However, such exotic stellar populations show a spectral energy distribution that peaks at short wavelengths, often in the near-ultraviolet $(N U V)$ or far-ultraviolet $(F U V)$. This makes the $F U V$ the ideal waveband to search for and study exotic sources such as BSs, CVs, blue and extreme horizontal branch (HB) stars, and LMXBs. In addition, main sequence (MS) stars and red giants, which make up the majority of the stars in a GC, are faint at such short wavelengths. As a result, FUV images tend to be considerably less crowded than optical images. So far, deep HST FUV observations to study the exotic stellar populations in GCs have been conducted in only a few clusters, namely 47 Tuc (Knigge et al. 2002, 2008), NGC 2808 (Brown et al. 2001; Dieball et al. 2005a; Servillat et al. 2008), M15 (Dieball et al. 2005b, 2007), NGC 1851 (Zurek et al. 2009, 2016; Maccarone et al. 2010), M80 (Dieball et al. 2010; Thomson et al. 2010) and NGC 6752 (Thomson et al. 2012).

Interacting CBs are also strong X-ray emitters, and indeed large numbers of X-ray binaries have been detected in a number of GCs with the Chandra X-ray telescope (e.g. Grindlay et al. 2001a,b; Pooley et al. 2002; Edmonds et al. 2003a,b; Heinke et al. 2003a,b; Hannikainen et al. 2005; Lugger et al. 2007; Elsner et al. 2008; Bogdanov et al. 2010; Cohn et al. 2010; Forestell et al. 2014). The combination of both X-ray and $F U V$ observations is thus an ideal approach to detect and study the interacting CBs in GCs.

NGC 6397 is the second closest GC to us, at $2.2 \mathrm{kpc}$ (Heyl et al. 2012), and is one of the best studied GCs. Hansen et al. (2013) suggested a metallicity of $[\mathrm{Fe} / \mathrm{H}]=-$ 1.8 dex and reddening of $\mathrm{E}_{B-V}=0.18$ mag. Husser et al. (2016) provided 3D spectroscopy of more than 12,000 stars in NGC 6397 and derived a mean metallicity of $[\mathrm{Fe} / \mathrm{H}]=-2.12$ dex, compared to $[\mathrm{Fe} / \mathrm{H}]=-2.02$ dex listed in the catalogue of Harris (1996, updated version 2010). Absolute proper motions and the cluster's orbit around the Galaxy have been measured by Kalirai et al. (2007) and Milone et al. (2006). Lind et al. (2011) investigated the chemical composition of RGB stars in NGC 6397 based on high-resolution VLT FLAMES spectra and suggested that the cluster contains a primordial $(\approx 30 \%)$ and a secondary $(70 \%)$ generation that formed from material enriched by the first generation. They further suggested that the current mass of the cluster is just $10 \%$ of its initial mass. Multiple generations were photometrically confirmed by Milone et al. (2012), who found that the MS in NGC 6397 splits into two components, suggesting the presence of two stellar generations. Heyl et al.
(2012) studied the cluster kinematics and estimate a cluster mass of $1.1 \times 10^{5} M_{\odot}$.

Richer et al. (2008) derived a deep optical CMD that nearly reaches the H-burning limit and showed, for the first time, the blue-turn and the truncation of the WD sequence in this cluster at $F 814 W \approx 28$ mag. The mass function (MF) agrees with a flat power-law or log-normal distribution, which in turn agrees with the predictions from N-body modelling presented in Hurley et al. (2008). The latter conclude that, in different fields in the cluster, the WD population did not change strongly due to dynamical processes, whereas the MF of the MS has been altered significantly by the cluster dynamics.

Comparing the observed CMD and WD cooling sequence with theoretical models, Torres et al. (2015) suggested an age of 12.8 Gyr, whereas Hansen et al. (2007) derived a younger (also WD based) age of 11.47 Gyr. Torres et al. (2015) used updated evolutionary tracks and suggested that this might be the reason for the difference in age compared to the findings from Hansen et al. (2007). These values bracket the MS turnoff age of 12 Gyr that has been suggested by both Gratton et al. (2003) and Winget et al. (2009). Comparing the CMDs of NGC 6397 with 47 Tuc and M4, Richer et al. (2013) found that NGC 6397's MS is much bluer (as expected because of its lower metallicity), but the location of the WD sequences agrees independent of metallicity. This is expected as WD atmosphere should be composed of only $\mathrm{H}$ and He. Thus, the location of the (bottom of the) WD sequence provides an ideal age indicator.

Cool et al. (1995) first searched for CVs in NGC 6397 using $R$ - and $H \alpha$-band $H S T$ WFPC imaging. They found three $\mathrm{H}$-alpha emission objects, all of them are also UV bright and ROSAT X-ray counterparts, making them good CV candidates. Chandra X-ray observations have been carried out by Grindlay et al. (2001a) and Bogdanov et al. (2010), detecting 79 X-ray sources within the half-mass radius of this cluster. Shara et al. (2005) used WFPC2 and STIS UV imaging and detected dwarf nova (DN) eruptions in two CVs that had previously been suggested to be magnetic systems (Grindlay et al. 2001b) and which are usually not expected to show DN eruptions. Shara et al. (2005) suggested that these two systems might be intermediate polars that occasionally undergo DN eruptions, which might explain the scarcity of DNs in GCs.

More recently, Cohn et al. (2010) carried out much deeper optical and $H \alpha H S T$ imaging, finding 69 optical counterparts to the $79 \mathrm{X}$-ray sources. Of these, 15 are CVs or CV candidates and two are MSPs. Optical emission of the bright and centrally concentrated CVs seems to be dominated by their accretion disks and secondaries, whereas the fainter CVs are optically dominated by their WD primary. A sequence of $24 \mathrm{He}$ WDs, parallel to the CO WD cooling sequence, has also been suggested to be present in the cluster's central region by Strickler et al. (2009).

Recently, Kamann et al. (2016) used integral field spectroscopic data and suggested that the surface brightness profile and velocity dispersion profile could either be explained by the presence of an intermediate-mass black hole with a mass of $600 M_{\odot}$ - which agrees with an earlier suggestion by deRijcke et al. $(2006)$ of $\approx 1300$ to $400 M_{\odot}$, based on radio continuum emission - or with a dark stellar component. 
Here, we present the results of a STIS FUV imaging analysis of the GC NGC 6397. We compare our data to WFC3 NUV and ACS optical data. The observations and data reduction are described in Sect. 2, and the analysis is presented in Sect. 3, including the UV and optical CMDs and a discussion of the various stellar populations. In Sect. 4 we present the $N U V$ lightcurves of the CVs and the MSP in our FUV field of view. The radial distribution of the various stellar populations that show up in our UV and optical CMDs are discussed in Sec. 5. Our results are summarized in Sect. 6.

\section{OBSERVATIONS AND DATA REDUCTION:}

\subsection{The STIS FUV Data}

The FUV data was obtained with the Space Telescope Imaging Spectrograph (STIS) on-board the HST using the FUVMAMA detector and the F25QTZ filter (program GO-8630, PI: M. Shara). The STIS FUV-MAMA instrument has a field of view of $25^{\prime \prime} \times 25^{\prime \prime}$ and a plate scale of $0.024^{\prime \prime} /$ pixel, and the central wavelength of the F25QTZ filter is at $1590 \AA$. Four F25QTZ images are available with exposure times of 1200, 2333, 865 and 600 seconds, resulting in a total exposure time of 4998 seconds.

As a first step we created a $F U V$ master image using the four pipeline-produced flat-fielded (i.e. calibrated) images. The flat-fielded images were aligned using the Tweakreg task from the Drizzlepac package running under PyRaf which computes the residual shifts between all the input images and the reference image (the first of our input images). Next, the AstroDrizzle task from the Drizzlepac package was used to combine all the aligned images and produce a geometrically corrected, deep master image.

\subsubsection{Source Detection}

In order to detect all potential sources, we used the Daofind task in the Daophot package (Stetson 1987) running under PyRAF to create an initial list of potential sources. We note that FUV images do not suffer from severe crowding, because MS stars and red giants, which make the majority of the stars in GCs, are not expected to be bright at such short wavelengths.

The coordinates of the initial source list were overplotted on the master image and carefully inspected by eye. Overall, the source detection routine Daofind worked well, but some of the detected sources appeared to be false detections around bright sources and PSF spikes and were consequently discarded. On the other hand, some faint sources could be detected by eye but had not been found by the detection routine and thus were added by hand to the final list, which comprises 192 sources.

\subsubsection{Aperture Photometry}

Aperture photometry was performed on the combined and geometrically-corrected FUV master image using Daophot (Stetson 1987) running under PyRAF. Since only about 200 sources are present in the $F U V$ master, i.e. the image is not very crowded, we set an aperture size of 8 pixels, with a small sky annulus of 8-12 pixels, so that for most sources no neighbouring source is within the sky annulus. We also allowed for a Gaussian re-centering of the input coordinates derived from Daofind.

However, an aperture radius of 8 pixels does not include $100 \%$ of the source flux, while on the other hand the small sky annulus means that not only background, but also source flux will be subtracted during sky subtraction. Thus, we need to correct for these two effects. Following Dieball et al. (2007), we picked a few isolated stars and measured their curve of growth (flux versus aperture radius) out to an aperture radius of 57 pixels, as well as their magnitude ratio using a small (8 - 12 pixel) and a large (60 to 70 pixel) sky radius. The flux correction factors turned out to be very small, with an aperture correction $\mathrm{ApC}=1.11 \pm 0.06$ and a negligible sky correction $(\mathrm{SkyC}=1.00 \pm 0.00)$. The magnitude of each source was then determined in the STMAG system as

STMAG $=-2.5 \times \log _{10}(\mathrm{CR} \times$ Photflam $\times \mathrm{ApC} \times \mathrm{SkyC})+\mathrm{ZPT}$

where Photflam is the inverse sensitivity of the filter taken from the image headers as $1.19004155 \times 10^{-16}$ ergs/seconds $/ \mathrm{cm}^{2} / \AA$ per counts/seconds, CR denotes the count rate of source, and ZPT $=21.1 \mathrm{mag}$ is the zeropoint for the STMAG system (also taken from the image header).

\subsection{The $N U V$ data}

The $N U V$ observations were obtained with the Wide Field Camera 3 (WFC3) UVIS/F225W camera/filter combination as part of program GO-11633 (PI: R. M. Rich). The WFC3/UVIS instrument has a field of view of $162^{\prime \prime} \times 162^{\prime \prime}$ and a plate scale of $0.04^{\prime \prime} /$ pixel. The data consist of 24 images with an exposure time of 680 seconds each, resulting in a total exposure time 16320 seconds.

The $N U V$ master image was created in the same way as the $F U V$ master image, using the pipeline produced, flatfielded images as input to Tweakreg and AstroDrizzle and default parameter settings. The FUV and NUV master images are shown in Figure 1.

\subsubsection{Creating the NUV Catalog}

As can be seen, the $N U V$ master image is considerably more crowded than the FUV master image. We therefor use the photometry package Dolphot which is a stellar photometry package adapted from HSTphot (Dolphin 2000) to search for sources and perform PSF-fitting photometry. In contrast to our FUV aperture photometry, the source finding and photometry routines are not performed on the deep NUV master image, but on the individual flat-fielded images, with the NUV master serving as a reference frame for the image coordinates. Photometry is performed on each individual flatfielded image using the WFC3 module within Dolphot, Jay Anderson's PSF library for the WFC3/UVIS F225W filter and the corresponding pixel area map ${ }^{1}$. The WFC3 module also includes built-in charge transfer efficiency (CTE) corrections and a photometric calibration to the VEGAmag

1 which are available on the Dolphot web-page: americano.dolphinsim.com/dolphot/ 


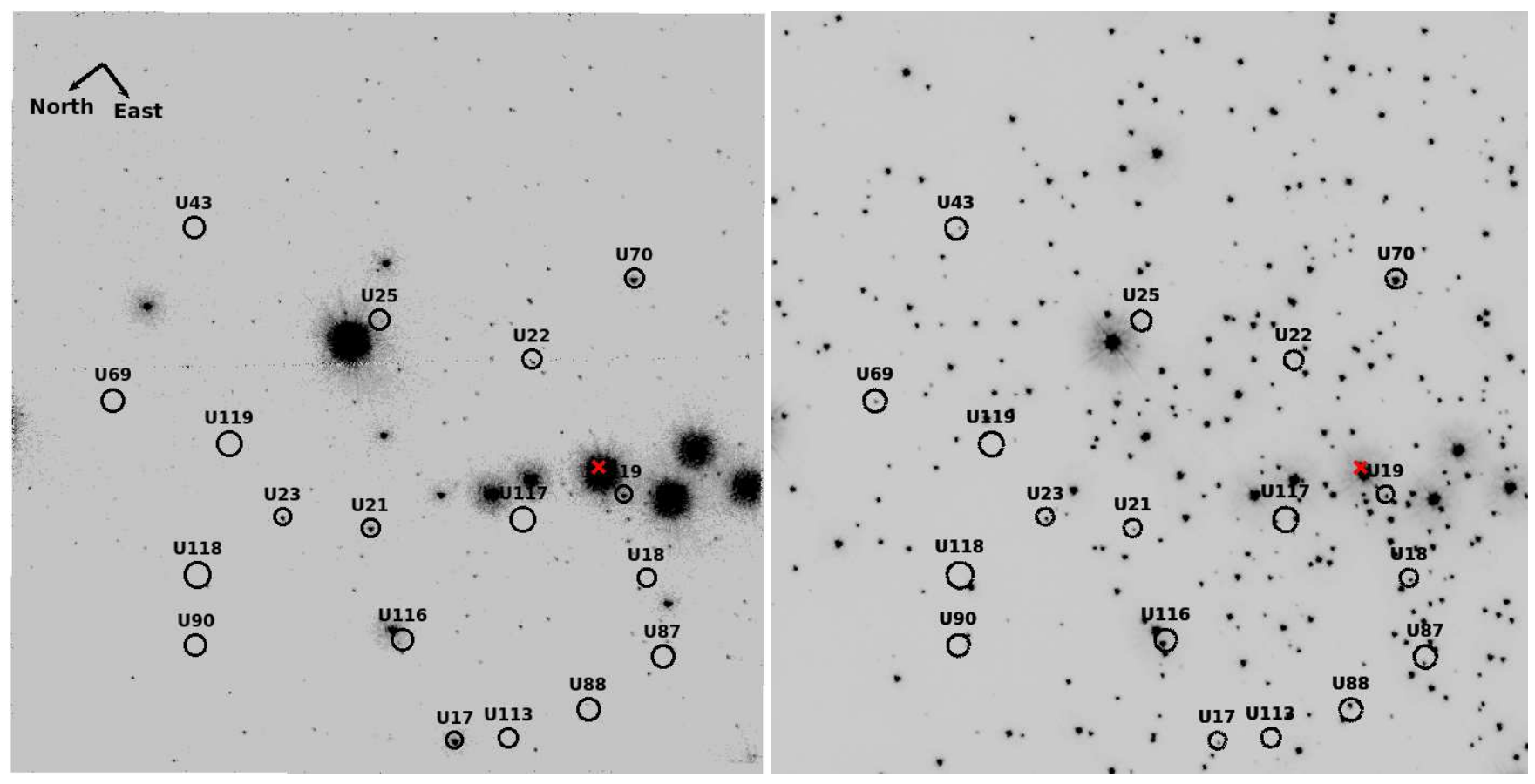

Figure 1. Left: The FUV master image of NGC 6397. Right: A subset of the $N U V$ master image covering the same field. Note that the full $N U V$ master image covers a much larger field of view $\left(162^{\prime \prime} \times 162^{\prime \prime}\right)$, whereas the area covered by the $F U V$ data is much smaller $\left(25^{\prime \prime} \times 25^{\prime \prime}\right)$. Both images are displayed on a logarithmic scale to bring out the faint sources. The locations of the X-ray sources are marked with their 95\% error circles and their X-ray denotation from Bogdanov et al. (2010) and Cohn et al. (2010). The centre of the cluster, taken from Cohn et al. (2010), is marked with a red cross and is close to the location of the X-ray source U 19 and nearly coincides with the location of a bright BS source.

system. Dolphot provides routines to mask all pixels that are flagged as bad in the data quality image, to multiply by the pixel area maps, and to align all the input images to the reference image using user-defined coordinate lists for each input and the reference image. For all these steps, we began with the recommended parameters and then optimized them such that the photometry was pushed as deep as possible. However, the three brightest stars located in the $F U V$ field of view are saturated in the $N U V$ data. The Dolphot output provides a magnitude from the individual images, and we used these to determine a mean magnitude for these three $N U V$ sources, however only for the sake of plotting them in the $F U V-N U V$ CMD, see Fig. 2. We caution the reader that these magnitudes are likely not the correct $N U V$ magnitude, as Dolphot returns an error flag for each individual measurement (too many saturated pixels). The three stars in question are marked in Fig. 2.

\subsection{Optical Catalog}

The main objective of this paper are the STIS FUV data and the FUV - NUV CMD (see Sect. 3.1). However, in order to find optical counterparts to our FUV sources, we also used some archival optical data obtained with the Advanced Camera for Surveys (ACS) in the F606W (corresponding to $V$-band) and F814W (corresponding to I-band) filters (program GO-10775,PI: A. Sarajedini) as part of the "ACS Survey of Galactic Globular Clusters" (Sarajedini et al. 2007). Data for each filter consist of one 1 second exposure and four 15 second exposures, i.e. a total exposure time of 61 seconds. The ACS/WFC instrument has a field of view of $202^{\prime \prime} \times 202^{\prime \prime}$ and a plate scale of $0.05^{\prime \prime}$ per pixel. We used the photometry catalogue provided via the "ACS Survey of Galactic Globular Clusters" web-page. The data and data reduction are described in detail in Sarajedini et al. (2007) and Anderson et al. (2008).

\subsection{The X-ray Catalog}

In order to find matches to known X-ray sources in our $F U V$ field, we used the catalog published in Cohn et al. (2010), who in turn used the X-ray data published in Bogdanov et al. (2010) and Grindlay (2006). Cohn et al. (2010) searched for optical counterparts to the 79 Chandra X-ray sources within the halfmass radius of NGC 6397, and identified $15 \mathrm{CVs}, 42 \mathrm{ABs}$, two MSPs, one active galactic nucleus, and one interacting galaxy pair candidates. In total, they found 69 optical counterparts to the 79 X-ray sources.

\subsection{Matching Catalogs}

All HST data used in this paper have been obtained at different times and with slightly different pointings. This has an impact on the world (RA, DEC) coordinates obtained from the images, i.e. even though the same source is measured, the derived coordinates typically differ slightly. For this reason, all coordinates first have to be transformed to a common coordinate system. We picked the $N U V$ master image $(\mathrm{F} 225 \mathrm{~W})$ as our overall reference image towards which all $F U V$ and optical (pixel) coordinates were transformed. The $N U V$ master image was chosen because it is not as crowded 
as the optical data, and therefore it was easiest to find stars in the $N U V$ master that could also clearly be identified in the optical and FUV data. We used 108 stars that were easily identified in the $N U V$ and $F U V$ master images to calculate the transformation coefficients using Geomap running under PyRAF. Next, the coordinate transformation was applied to the whole FUV coordinate list using the Geoxytran task running under PyRAF to transform them to the $N U V$ reference coordinate system. For the optical coordinate transformation, we used 136 stars that can be clearly identified in the $N U V$ master and the ACS optical catalogue. Again, we first used Geomap to work out the coordinate transformation, and applied Geoxytran to the whole optical coordinate list to transform the ACS coordinates to our $N U V$ reference system.

In order to find possible matches to the known X-ray sources, we also transformed the X-ray coordinates taken from Table 1 in Cohn et al. (2010) to the NUV master coordinate system. Since Cohn et al. (2010) tied their astrometry to the USNO UCAC2 catalogue, we used 30 of the 36 USNO UCAC2 stars that are located within the NUV master field of view and for which a $N U V$ counterpart could be clearly identified. Again, we use Geomap and Geoxytran to work out the coordinate transformation from UCAC2 to our $N U V$ reference frame. We then applied this transformation to the X-ray source coordinates listed in Table 1 in Cohn et al. $(2010)^{2}$.

After transforming all coordinates into a common $(N U V)$ coordinate system, we chose a maximum tolerance radius of $2 N U V$ pixels to search for counterparts in the $F U V$ and optical.

We also searched for $F U V$ and $N U V$ counterparts to $\mathrm{X}$-ray sources within the $95 \%$ positional uncertainty radius given in Bogdanov et al. (2010), see Fig. 1. All possible FUV and NUV counterparts are listed in Table 1, see Sect. 3.6 below.

\subsection{False Matches}

The source density in the $N U V$ and optical images is high and these images are much more crowded than the FUV image. Thus we might expect a few false matches among our FUV -NUV and $N U V$-optical pairs. The number of these spurious pairs depends on the number of sources in the $F U V$ (192 sources), NUV (416 sources) and optical (678 sources) in the $F U V$ field of view, our chosen matching tolerance radius (2NUV pixel), and the number of sources actually matched (182 FUV/NUV pairs, 175 FUV/optical and 407 $N U V$ /optical pairs). Following the approach in Knigge et al. (2002), we expect fewer than one false match (0.14) among the $F U V-N U V$ pairs, and also fewer than one false match (0.16) among the NUV-optical pairs.

\footnotetext{
2 Note that we first transformed all coordinates from the world coordinate system to the $N U V$ master image physical system, i.e. pixel coordinates, and all transformations are applied to the corresponding pixel coordinates so that all coordinates which are used for matching are in the $N U V$ image (physical) reference frame.
}

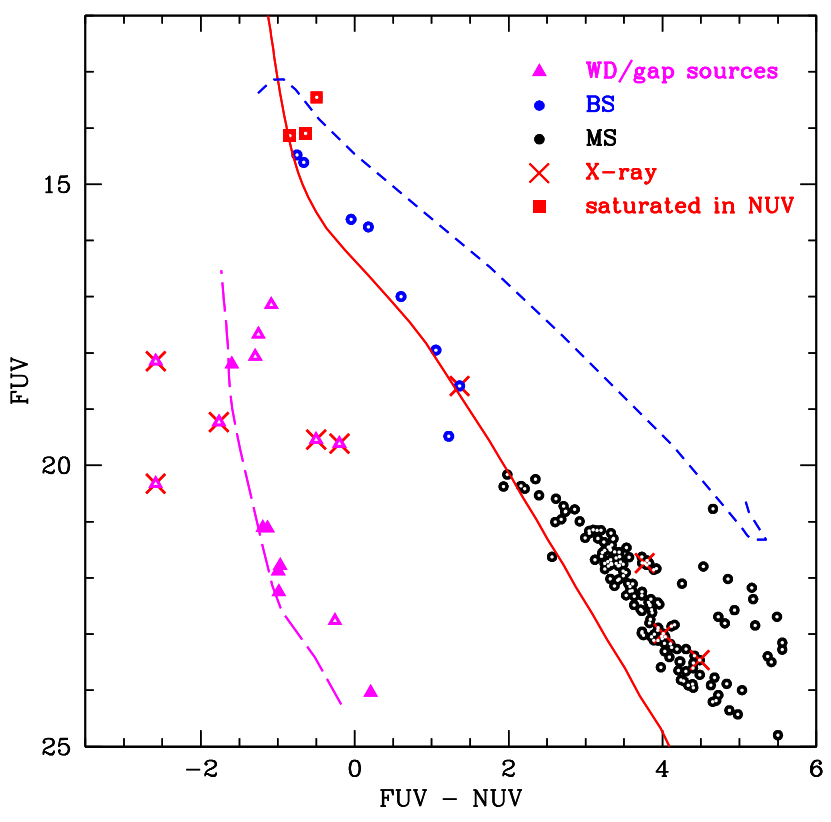

Figure 2. $F U V$ vs. $F U V-N U V$ CMD. For orientation purpose, we also show the synthetic ZAHB (blue, short-dashed line), ZAMS (red, solid line) and WD cooling sequences (magenta, longdashed line). Sources with optical counterparts are marked with open symbols. Large black and blue dots/circles denote MS and BS stars, respectively. The three brightest sources are saturated in the $N U V$ and are marked with red squares, their true $N U V$ magnitudes are likely brighter and hence their colour might also be redder. Sources that are possible X-ray counterparts are marked with red crosses, and WD/Gap sources, which include CVs, are marked with magenta triangles.

\section{DATA ANALYSIS:}

In this section, we discuss the CMDs and the various stellar populations that show up, including WDs, CVs, HB stars, BSs and X-ray counterparts.

\subsection{The $F U V-N U V$ CMD}

The FUV - NUV CMD is plotted in Fig. 2. For orientation purpose, we also show the location of a theoretical zero-age MS (ZAMS, red line), a zero-age horizontal branch (ZAHB, blue short-dashed line), and a WD cooling sequence (magenta long-dashed line). In order to create a ZAMS, we used the fitting formulae provided by Tout et al. (1996) to estimate appropriate stellar parameters. We then interpolated on the grid of Kurucz models and folded the resulting synthetic spectra through the response of the appropriate filter+detector combinations using Synphot running under PyRAF. For the ZAHB track, the grid of oxygenenhanced ZAHB models provided by Dorman (1992) was used to generate a set of models closest to the cluster metallicity. Again, the corresponding $F U V$ and $N U V$ magnitudes were computed by interpolating on the Kurucz model grid and folding through the corresponding filter transmission curves using Synphot. We used standard cluster parameters of $E_{B-V}=0.18 \mathrm{mag}$, a distance of $2.2 \mathrm{kpc}$, and a metallicity 
of $[\mathrm{Fe} / \mathrm{H}]=-2.02 \mathrm{dex}$ (Harris 1996), which gives a good fit of the theoretical sequences to the underlying data.

We can see a relatively tight sequence of sources (small black data points/circles) reaching from the red, faint corner at $F U V \approx 24$ mag towards bluer and brighter magnitudes, with only eleven sources (blue circles and red squares) brighter than $20 \mathrm{mag}$ in the $F U V$ along the ZAMS. The faint, red sequence consists mostly of MS stars, whereas the eleven bright sources agree with being BSs or possibly blue HB stars. The three brightest sources are saturated in the $N U V$ and we assign a mean magnitude based on the magnitudes in the individual input images only for plotting purpose. Their true $N U V$ magnitude is likely brighter and as a consequence they might be redder. At first glance, it is not clear whether these three sources are bright BSs or possibly blue HB stars.

Sixteen sources are located on the blue side of our ZAMS. This is where we expect WDs and gap sources (i.e. sources that are located in the gap between the ZAMS and the WD cooling sequence, see Knigge et al. 2002). For comparison, we computed a theoretical WD sequence by interpolating on the Wood (1995) grid of theoretical WD cooling curves, adopting a mean WD mass of $0.55 M_{\odot}$. The models were translated to the observational plane by carrying out synthetic photometry with PySynphot, using a grid of synthetic DA WD spectra provided by Gänsicke et al. (1995) with temperatures ranging from $60,000 \mathrm{~K}$ to $10,000 \mathrm{~K}$. It seems that the hottest WDs in our $F U V-N U V$ CMD have temperatures around 48,000 K, corresponding to $F U V \approx$ $17 \mathrm{mag}$, whereas the coolest WD (candidates) are around $F U V \approx 24 \mathrm{mag}$, corresponding to $T_{\text {eff }} \geq 10,000 \mathrm{~K}$.

\subsection{Optical CMD}

As can be seen in Fig. 2, the ZAHB and ZAMS cross at $F U V \approx 13$ mag. Based on just the $F U V-N U V$ CMD, it is not immediately clear whether the three brightest sources are BSs or $F U V$-bright blue HB stars. In order to address this issue, we searched for optical counterparts to all FUV sources which helps to distinguish between the various stellar populations.

The optical CMD is based on the ACS survey (Sarajedini et al. 2007) and plotted in Fig. 3. Again, BSs are marked in blue, the three sources saturated in the NUV are marked with red squares, and WD/gap sources are denoted with magenta triangles. Counterparts to X-ray sources are marked with red crosses. All FUV sources that have an optical counterpart, and vice versa all optical sources that have a $F U V$ counterpart, are indicated with open symbols in Fig. 2 and Fig. 3. We also overplot the theoretical sequences discussed in Sect. 3.1, which fit the underlying CMD well. However, again it is not conclusive whether the three sources marked with red squares are the brightest BSs in the field of view, or blue HB stars. Note that we only show sources that are located within the $F U V$ field of view. Thus, for comparison, we show the optical CMD from the full ACS field of view in Fig. 4. Again, we marked those optical sources with a $F U V / N U V$ counterpart with open symbols. Based on this CMD, it appears that the brightest of the three sources saturated in the $N U V$ is indeed a blue HB star. The other two sources are most likely bright and blue BSs, but could also be fainter (and slightly redder) extreme blue HB (EHB)

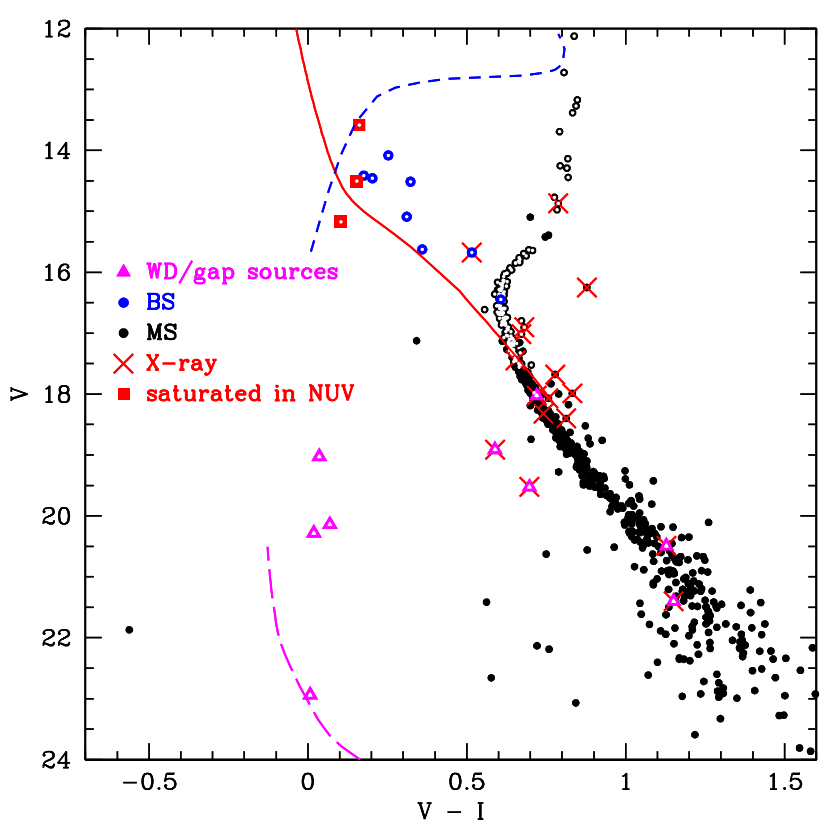

Figure 3. Optical CMD of NGC 6397, data taken from the ACS Survey of Galactic Globular Clusters (Sarajedini et al. 2007). The symbols are the same as in Fig. 2, i.e. WDs are marked with magenta triangles, BSs with blue circles (selected from the $F U V-$ NUV CMD in Fig. 2), the three sources saturated in the NUV are marked with red squares, and possible counterparts to Xray sources are denoted with red crosses. All optical sources that have a $F U V$ counterparts are marked with open symbols. We also overplot the same theoretical sequences as in Fig. 2. Note that this CMD covers the FUV field of view only, not the full ACS field of view.

stars. A classification of these three sources was not possible based on the $F U V-N U V$ CMD alone.

\subsection{Horizontal Branch Stars}

Only one, possibly up to three HB stars seem to be located within the $F U V$ field of view which covers only the inner $25^{\prime \prime} \times 25^{\prime \prime}$ of the cluster, see Fig. 2 and 3 . Thus, HB stars seem to be rare in the very core region of this cluster. This is likely an effect of the dynamical evolution of this cluster and mass segregation, i.e. more massive stars travel towards the cluster centre, but less massive stars, like the HB stars which typically have masses of $\approx 0.5 M_{\odot}$, are pushed out of the dense cluster centre (see e.g. Dieball et al. 2010, who derived an average mass of $\approx 0.6 M_{\odot}$ for the HB population in $\mathrm{M} 80)$.

$44 \mathrm{HB}$ stars are located within the full ACS field of view, which covers $202^{\prime \prime} \times 202^{\prime \prime}$ (and indeed somewhat more, because four images were taken per filter during the ACS observations, shifted by $\approx 4^{\prime \prime}$ in $\mathrm{x}$ and $\mathrm{y}$ direction). Assuming an even distribution of the HB stars across the field (i.e. ignoring the concentration of stars towards the cluster centre as well as effects of mass segregation), and scaling this number to the field of view covered by the STIS FUV observation, we would expect less than one HB star.

Next, we try to take the central concentration into ac- 


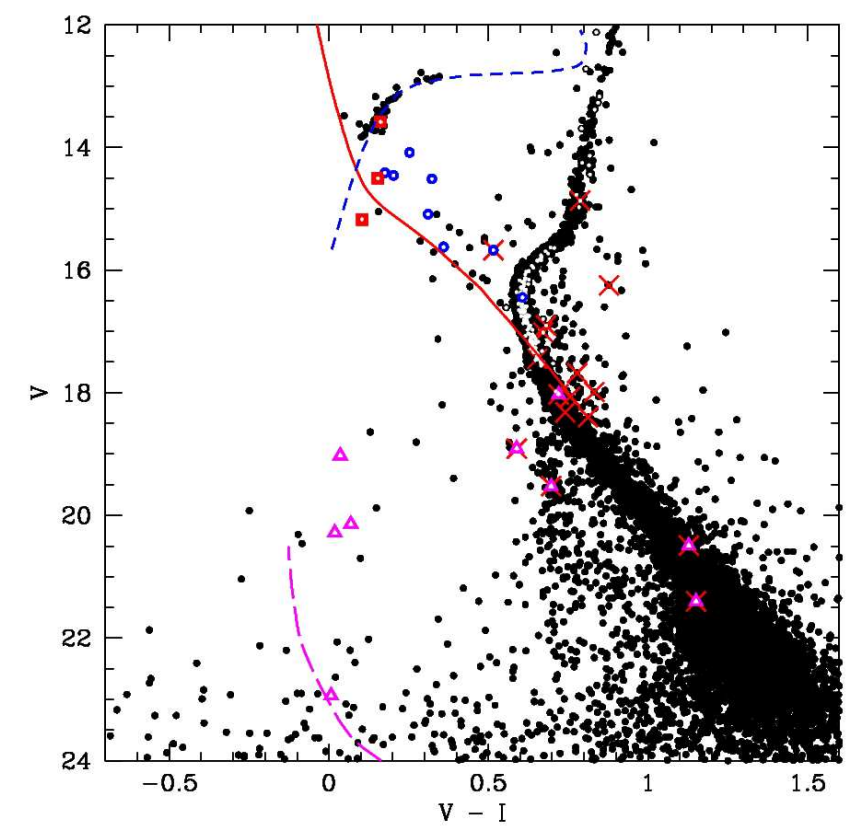

Figure 4. The same as Fig. 3, but for the full optical field of view. The symbols and theoretical tracks are the same as in Fig. 2 and 3.

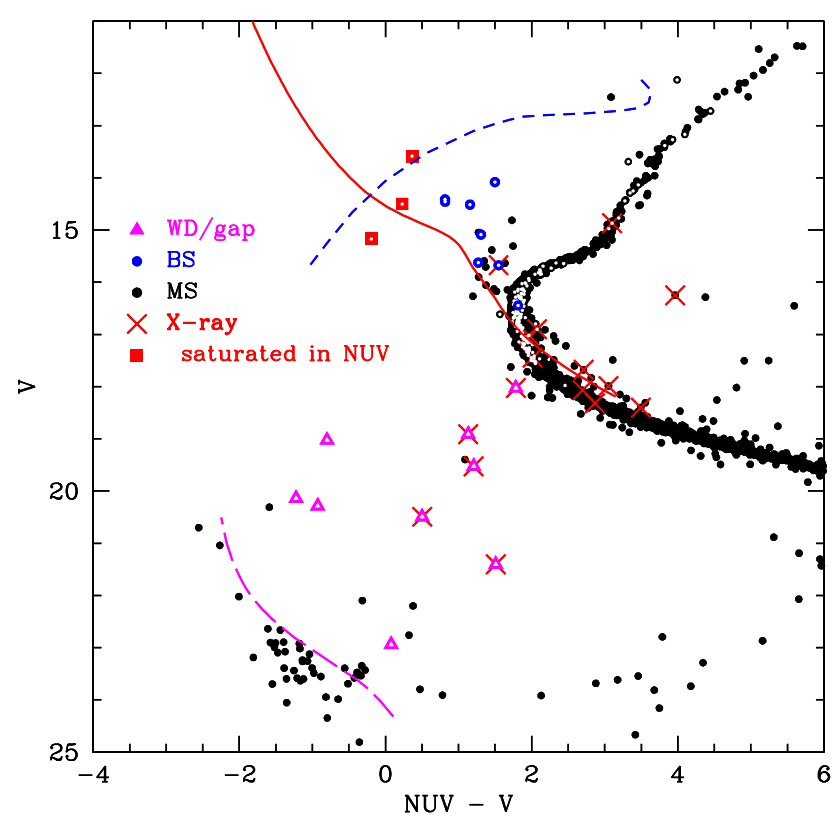

Figure 5. $N U V-V \mathrm{CMD}$, note that this CMD covers a larger area than the $F U V$ master image, but the $N U V$ and optical images do not fully overlap. The symbols and theoretical tracks are the same as in Figs. 2, 3 and 4. A WD sequence can be clearly seen. count by assuming that HB stars have a distribution more similar to the MS stars. We find 3136 MS stars within the full ACS field of view, out of those, 588 (18.7\%) are located within a $19.8^{\prime \prime}$ (corresponding to $500 \mathrm{NUV}$ pixel) radius around the cluster centre, 83 stars $(2.6 \%)$ within a $5.5^{\prime \prime}$ (130 NUV pixel) radius, and $345(11 \%)$ within the $F U V$ field of view (these numbers are based on the ACS optical CMD, down to $V=20 \mathrm{mag}$, see Fig. 4, and see also Sect. 5). Assuming that the HB stars follow a distribution similar to the MS stars, this translates to $8_{-5.9}^{+12.7} \mathrm{HB}$ stars within the 500 pixel radius (we found six $\mathrm{HB}$ stars), $1_{-1}^{+7.9} \mathrm{HB}$ star within the 130 pixel radius (we found none), and about $5_{-4.2}^{+11} \mathrm{HB}$ stars within the full FUV field of view (we found one HB star), where the errors are the $3 \sigma$ confidence limits taken from Gehrels (1986, Tables 1 and 3). The probability of finding one or less HB stars within the FUV field of view is $3.2 \%$ according to Bernouille statistics. This is not significant.

Thus, the small number of HB stars found in our $F U V$ field of view could as well simply be a statistical effect.

\subsection{Blue Stragglers}

Blue stragglers are peculiar stellar systems, since they are not following standard single star evolution. They are found in all GCs in the Galaxy, and are located above the MS turnoff in the CMDs but close to the ZAMS, i.e. they occupy a region in the CMD that should be devoid of stars. Their CMD location indicates that they are more massive than MS stars, and they also seem to be more centrally concentrated (e.g. Ferraro et al. 2003), another indication of their higher than average mass. The formation mechanism of BSs is still a matter of debate, but there are two generic scenarios for these systems: BSs might form from the collision of two MS stars resulting in a rejuvenated and more massive (MS) star (Hills \& Day 1976), or they might have formed via the evolution of primordial binaries (McCrea 1964).

A well-defined trail of eleven stars above the MS turnoff along (but slightly redder than) the ZAMS can be seen in Fig. 2 and 3, marked in blue (and red for the three sources saturated in the $N U V$ ). This is the expected location of the BS population. As discussed above in Sec. 3.3, one to three of the brightest of these stars might be blue HB stars.

We can identify $29 \mathrm{BSs}$ in the full optical CMD, however, only ten of those are located inside the field of view of our $F U V$ data (assuming that only the brightest $F U V$ source is a HB star). Nine of our FUV selected BSs are optically bright, the remaining $F U V$ bright BS is optically faint and located in the MS turn-off region. Its $F U V$ excess might be explained with a WD companion which contributes flux to the FUV (see Gosnell et al. 2015, who found BS-WD binaries in the old open cluster NGC 188)

\subsection{White Dwarfs/Gap Sources}

A population of 16 sources close to the theoretical WD cooling sequence can be seen in our $F U V-N U V$ CMD in Fig. 2. The scatter of sources around the bright, hot part of the WD sequence is large, so we cannot distinguish single WDs, He WDs or binary systems based on the FUV-NUV CMD alone. However, CVs are interacting binaries containing a WD accreting from a low-mass companion star. They are 
expected to be located between the WD cooling sequence and the ZAMS.

The NUV $-V$ CMD is displayed in Fig. 5. Again, we show the theoretical sequences, and we can clearly see a trail of blue and faint data points that agree nicely with the theoretical WD sequence. Sources that have a $F U V$ counterpart are marked with open symbols. Only nine WD/gap sources have a $F U V, N U V$ and optical counterpart. In the $N U V-V$ $\mathrm{CMD}$, they are all located between the WD cooling sequence and the MS, whereas in the $F U V-N U V$ CMD, three of these sources are located on the blue side of the FUV WD cooling sequence. These three sources are also X-ray sources, suggesting that they are interacting binaries, i.e. CVs, and indeed their location in the $N U V-V$ CMD supports this classification.

\subsection{X-ray counterparts}

CVs are also X-ray sources, so we searched for counterparts to known X-ray sources in the inner area of NGC 6397, listed in Cohn et al. (2010). In total, there are 18 X-ray sources in the FUV field of view. The $95 \%$ positional uncertainty radius of these X-ray sources were taken from Bogdanov et al. (2010). The position of the X-ray sources are marked in Fig. 1 and we searched for counterparts within their $95 \%$ confidence radii.

Nine out of the 18 X-ray sources had both a FUV and a corresponding $N U V$ counterpart, namely U17, U19, U21, U22, U23, U70, U88, U116 and U117. Five of these, the counterparts to U17, U19, U21, U22, and U23, are located in the WD or gap area in the $F U V-N U V$ CMD, which makes all of them good CV candidates. Indeed, Cohn et al. (2010) also suggested that these sources are CVs. In their $H \alpha-R$ CMD (their Fig. 3), these five sources are bright $H \alpha$-excess sources, i.e. they are optically bright CVs. Their optical counterparts in Fig. 3 are located on or slightly blueward of the MS and are all fainter than $V<18$ mag.

The counterpart to U70 is a bright $F U V / N U V$ source and is located on the ZAMS in the BS area above the MS turnoff in our FUV - NUV CMD. This source was also identified as a BS in Cohn et al. (2010). This is certainly an interesting and peculiar object that deserves future observation.

The remaining possible counterparts to U88, U116 and U117 are all located on the red side of the MS in all CMDs, i.e. they are likely AB stars. This agrees with the classification of Cohn et al. (2010, their Table 1 and Fig. 4). The $U V$ counterpart to U88 is located just at the rim of its error circle. For U116 and U117, two NUV sources are located within their error circles. For U116, only the brighter of the two $N U V$ sources has a $F U V$ counterpart which places this source on the MS in the $F U V-N U V$ CMD, marked with a red cross. Both $N U V$ sources have an optical counterpart (marked with red crosses in the optical and NUV-optical CMDs), however, one is located on the RGB and the other on the optical MS. Cohn et al. (2010) identified a MS star as the counterpart to U116, thus, the brighter source on the RGB might not be the real counterpart but the fainter source on the MS (which has, however, no FUV match). For U117, both of the two NUV sources are close to the rim of the X-ray error circle. Only one has a FUV counterpart, but both have an optical counterpart which are both marked with a cross in the optical CMD in Fig. 3. They are located close to each other on the MS, but we cannot decide which might be the real counterpart based on the CMDs alone.

X-ray source U25 only has a FUV counterpart but no $N U V$ source was detected. However, there seems to be a faint smudge at the position in the $N U V$ master image, but no magnitude could be assigned. Since this source does not appear in our $F U V-N U V \mathrm{CMD}$, we cannot attempt any further classification, however Cohn et al. (2010) classified $\mathrm{U} 25$ as a possible CV.

U18 was classified as a likely MSP by Cohn et al. (2010) and Bogdanov et al. (2010). We can clearly detect a $N U V$ counterpart, however, the $F U V$ master image only shows a faint smudge at the position of the $N U V$ source.

Also sources U43, U69, and U87 only had a NUV counterpart, but no FUV counterparts could be detected. Thus, we cannot classify these sources, however Cohn et al. (2010) classified all three sources as ABs. For source U43, two NUV sources and corresponding optical counterparts are located within its X-ray error circle. Both sources are located on the MS in the optical CMD. The brighter $N U V$ source is closer to the centre of the error circle and thus might be the true counterpart.

No $U V$-counterparts have been found for the X-ray sources U90, U113, U118 and U119. The NUV master image shows a faint smudge within the U90 error circle, but no measurement was returned by our Dolphot photometry. U90 is classified as an AB, and U118 is classified as a MSTO star in Cohn et al. (2010), but sources U113 and U119 still need to be classified.

The X-ray sources with a $F U V$ and/or $N U V$ counterpart that are located inside the $F U V$ field of view are listed in Table 1.

\section{$4 N U V$ VARIABILITY}

CVs are known to show all sorts of variable behavior in their light curves (Warner 2003). Since only four images are available in the $F U V$ data set, we used the $N U V$ data, which comprised 24 images, to construct light curves for the five CVs in our FUV - NUV CMD - U17, U19, U21, U22, and U23 - and for the $N U V$ counterpart to the MSP U18. The NUV observations were taken on the 9th and 11th of March 2010, and all 24 images were obtained with the same exposure time of 680 seconds. All the individual $N U V$ magnitudes were determined with Dolphot. Fig. 6 shows the light-curves for all these six sources. We plot the difference between observed magnitude and mean magnitude, $\Delta N U V=\overline{N U V}-N U V_{\text {obs }}$, against observing time in MJD. Note that the magnitudes assigned to the source (and hence plotted in the CMDs in Fig. 2 and 5) is the weighted mean (see also Table 1), which is slightly different to the mean given in the right panel and used to obtain $\triangle N U V$.

All light curves show brightness variations of a few tenths of a magnitude on short timescales of minutes. This is typical of the aperiodic "flickering" seen in CVs.

The light curves of the $N U V$ counterparts to the $\mathrm{X}$ ray sources U17, U19 and U22 all show variation of up to $\approx$ $0.5 \mathrm{mag}$ (semi-amplitude) on short timescales, indicating the typical flickering variability characteristic of CVs. Towards the end of the fourth and the beginning of the fifth observing 
Table 1. FUV and/or $N U V$ counterparts to the X-ray sources that are located within the $F U V$ field of view. The $V$ and $I$ magnitudes are taken from the optical catalogue provided by the ACS survey of Galactic Globular Clusters, see Sarajedini et al. (2007). The last column gives our classification as a CV, BS or MS source, based on the position of the counterpart in the $F U V-N U V$ and/or optical CMD. The ${ }^{1}$ denotes the classification derived in this paper, a ${ }^{*}$ denotes the classification given in Cohn et al. (2010), which agrees well with ours. See the text for details.

\begin{tabular}{cccccccccc}
\hline $\mathrm{Id}_{\text {Xray }}$ & $F U V$ & $\Delta F U V$ & $N U V$ & $\Delta N U V$ & $F U V-N U V$ & $V$ & $V-I$ & $N U V-V$ & type \\
\hline U17 & 18.147 & 0.016 & 20.732 & 0.005 & -2.585 & 19.525 & 0.697 & 1.207 & $\mathrm{CV}^{1, *}$ \\
$\mathrm{U} 18$ & - & - & 20.214 & 0.004 & - & 16.250 & 0.878 & 3.964 & $\mathrm{MSP}^{*}$ \\
$\mathrm{U} 19$ & 19.542 & 0.038 & 20.043 & 0.004 & -0.501 & 18.911 & 0.589 & 1.132 & $\mathrm{CV}^{1, *}$ \\
$\mathrm{U} 21$ & 19.231 & 0.026 & 20.996 & 0.006 & -1.765 & 20.493 & 1.128 & 0.503 & $\mathrm{CV}^{1, *}$ \\
U22 & 20.326 & 0.043 & 22.913 & 0.017 & -2.587 & 21.403 & 1.151 & 1.510 & $\mathrm{CV}^{1, *}$ \\
U23 & 19.612 & 0.031 & 19.810 & 0.003 & -0.198 & 18.026 & 0.720 & 1.784 & $\mathrm{CV}^{1, *}$ \\
U25 & 21.475 & 0.128 & - & - & - & - & - & - & $\mathrm{CV}^{*}$ \\
U43 & - & - & 21.040 & 0.006 & - & 17.989 & 0.832 & 3.051 & $\mathrm{MS}^{1}, \mathrm{AB}^{*}$ \\
U43 & - & - & 21.186 & 0.006 & - & 18.319 & 0.741 & 2.867 & $\mathrm{MS}^{1}(?)$ \\
U69 & - & - & 20.766 & 0.005 & - & 18.070 & 0.757 & 2.696 & $\mathrm{AB}^{*}$ \\
U70 & 18.587 & 0.019 & 17.224 & 0.001 & 1.363 & 15.676 & 0.516 & 1.548 & $\mathrm{BS}^{1}, \mathrm{AB}^{*} \mathrm{BS}^{*}$ \\
U87 & - & - & 20.389 & 0.004 & - & 17.679 & 0.778 & 2.710 & $\mathrm{AB}^{*}$ \\
U88 & 23.464 & 0.182 & 18.980 & 0.002 & 4.484 & 16.904 & 0.681 & 2.076 & $\mathrm{MS}^{1}, \mathrm{AB}^{*}$ \\
U116 & 21.741 & 0.120 & 17.972 & 0.001 & 3.769 & 14.871 & 0.788 & 3.101 & $\mathrm{MS}^{1}(?)$ \\
U116 & - & - & 21.894 & 0.010 & - & 18.400 & 0.813 & 3.494 & $\mathrm{AB}^{*}$ \\
U117 & - & - & 19.460 & 0.003 & - & 17.447 & 0.653 & 2.013 & $\mathrm{AB}^{*}$ \\
U117 & 22.992 & 0.147 & 18.981 & 0.002 & 4.011 & 17.017 & 0.672 & 1.964 & $\mathrm{MS}^{1}, \mathrm{AB}^{*}(?)$ \\
\hline
\end{tabular}

epoch, U17 and U19 show some brightening. However, the epoch in between our fourth and fifth epoch is not covered by the $N U V$ observations, so we cannot tell whether the sources were indeed brighter in between the fourth and fifth epoch or not. Shara et al. (2005) suggested that the CVs U17 (CV3 in Shara et al. 2005, Grindlay et al. 2001b) and U19 (CV2) underwent DN eruptions. U17 (CV3) was seen to brighten in optical WFPC2 data, but not in the STIS FUV data available at that time. However U19 (CV2) was seen to brighten in the FUV F25SRF2 data, compared to the FUV F25QTZ data. We do not observe any DN outbursts in our $N U V$ data.

U21 also shows small amplitude variations on short timescales. Cohn et al. (2010) reported that U21 had the largest brightening in their $\mathrm{H} \alpha$ variability study. The NUV counterpart is brighter by about one magnitude at the beginning of the third observing epoch, but no further trend is apparent.

U18 and U23 also show somewhat smaller variations of $\approx 0.2 \mathrm{mag}$ (semi-amplitude). U23 was classified as a CV, and the light-curve shows the typical flickering behaviour. U18 is a likely MSP, and its light-curve shows flickering behaviour, but other than that we do not see any periodic variability.

\section{RADIAL DISTRIBUTIONS OF THE STELLAR POPULATIONS}

\subsection{The Cluster Center}

In order to study the radial distribution of the stellar populations that show up in our $F U V-N U V$ and $N U V-V$ CMDs, we used the cluster centre suggested by Cohn et al. (2010), at $\mathrm{RA}=17^{\mathrm{h}} 40^{\mathrm{m}} 42.17^{\mathrm{s}}, \mathrm{DEC}=-53^{\circ} 40^{\prime} 28.6^{\prime \prime}$. We transformed these coordinates to our NUV reference frame, using the same transformation that was used to bring the X-ray coordinates to the $N U V$ reference frame (see section 2.5).

\subsection{Radial Distribution}

Unfortunately, the $F U V$ observations were not centred on the cluster centre, see Fig. 1. Only a circular area with a $5.5^{\prime \prime}$ radius is fully covered by the $F U V$ data, larger annuli are only partially covered. However, the $N U V$ and optical data fully cover a larger circle of $19.8^{\prime \prime}$ radius. Therefore we used the $N U V-V$ CMD in Fig. 5 to select the different stellar populations, i.e. MS stars, BSs, and WDs. We also include sources that are missing from the $N U V-V$ CMD but are detected in the FUV data. These include one BS which is above the MS turnoff in the $F U V-N U V$ CMD but within the MS in the optical CMD, and seven WDs that can be seen in the FUV - NUV CMD but have no optical counterpart.

HB stars are located within the inner 19.8", but only one has a $N U V$ (and $F U V$ ) counterpart (the brightest FUV source, saturated in the $N U V$, see Sect. 3.4). Thus, we selected the HB stars based on the optical CMD in Fig. 4.

For the radial distribution of the X-ray sources, we used the catalogue (and coordinates) given in Cohn et al. (2010). A subset of the X-ray sources are classified as interacting X-ray binaries in Cohn et al. (2010). These include mostly CVs, but also one qLMXB and one MSP (source U18 discussed above) are located within the annulus covered by our data. The FUV field of view contains $18 \mathrm{X}$-ray sources, but only 14 have a $F U V$ and/or $N U V$ counterpart, five of those are classified as CVs. U25 is also classified as a CV but only has a $F U V$ and no $N U V$ counterpart. U18 is a MSP for which we detected the $N U V$ counterpart, but no FUV match.

The number of sources found in the full FUV field of view, the inner 5.5" and the inner 19.8" are given in Table 2 . Note that the stellar populations and the corresponding numbers for the full $F U V$ field of view were selected based on the $F U V-N U V$ CMD, but the number of sources within the inner 5.5" and 19.8" were selected based on the $N U V-V$ CMD, and the optical CMD for the HB stars, as outlined above. The number of all X-ray sources and of the CVs within the inner 5.5" and 19.8"was selected from the 


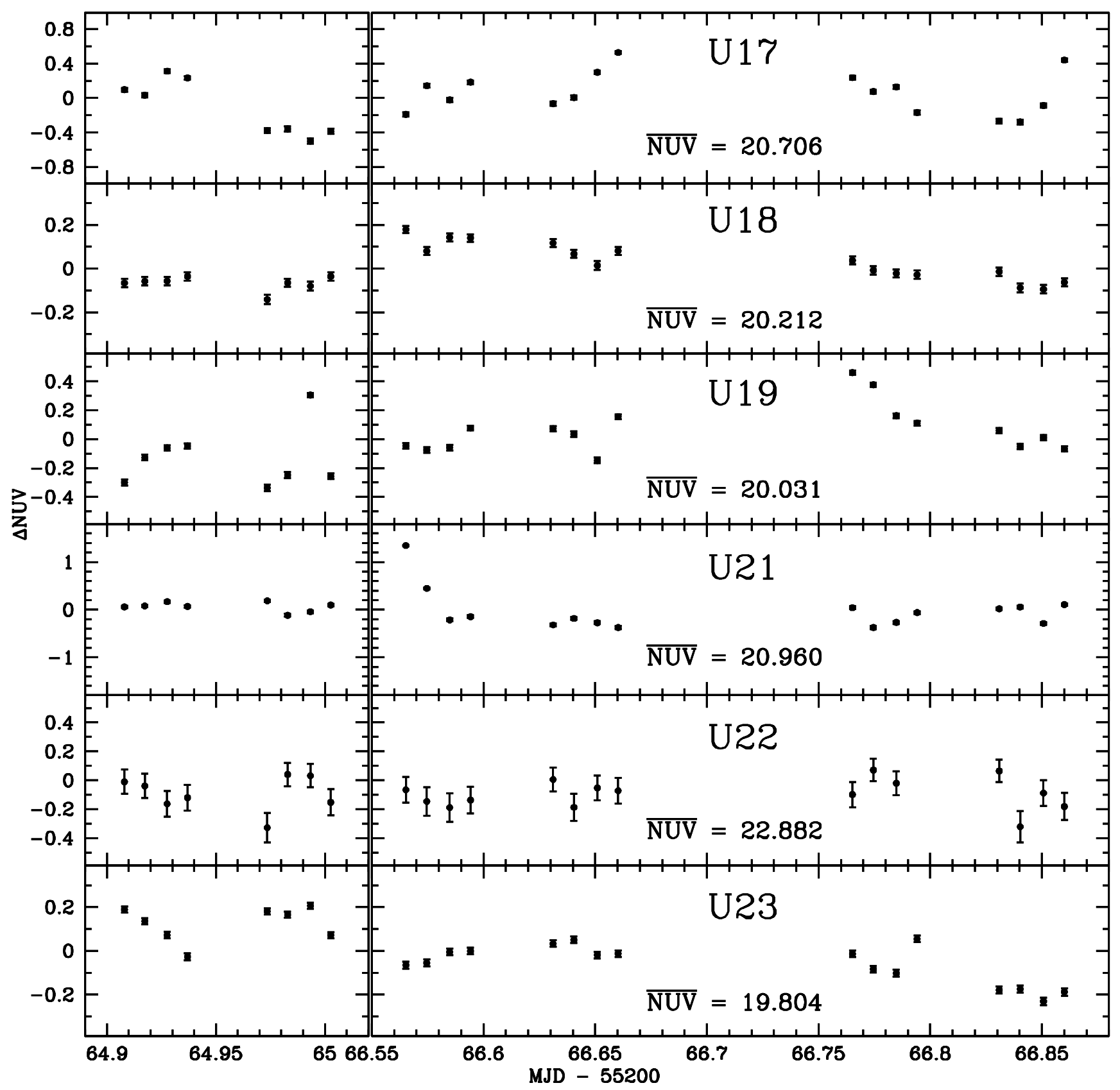

Figure 6. Light curves of the five $N U V$ counterparts to the CVs U17, U19, U21, U22 and U23, and the MSP U18 (second panel from the top). The $N U V$ observations were carried out on the 9th (first two epochs) and 11th (last four epochs) of March 2010. The NUV variation from the mean $N U V$ magnitude is plotted versus the modified Julian date (MJD). Their mean (average) $N U V$ magnitude is indicated. See the text for details.

catalogue and coordinates presented in Cohn et al. (2010), irrespective of them having a counterpart in any of the other wavebands or not. The CV sample is selected from the X-ray catalogue and also includes the MSP and the qLMXB, see above.

Fig. 7 shows the radial distribution of all the stellar populations discussed above. BSs are the most centrally concentrated population in both circular areas, while the HB stars are the least centrally concentrated populations. In fact, HB stars are absent in the inner circle with 5.5" radius. The CVs (six CVs and the MSP U18) are concentrated towards the cluster centre. Pooley \& Hut (2006) suggested that the CV population in GCs must have a large dynamically formed component. Based on their location in the core of the GC, these six CVs might have formed via dynamical processes. On the other hand, their location might also be the result of mass segregation, i.e. more massive sources, like CVs and BSs, travel to towards the cluster core.

We applied the Kolmogornov-Smirnov (KS) test to compare all stellar populations, see Table 3 . The KS test returns 
Table 2. Number of sources associated with each stellar population. Col. 2 lists the number of sources within the full $F U V$ field of view and based on the $F U V-N U V$ CMD only. Col. 3 lists the numbers within a $5.5^{\prime \prime}$ circular area centred on the cluster centre and fully covered by all wavebands, and Col. 4 denotes the number of sources within the inner $19.8^{\prime \prime}$ covered by the $N U V$ and optical data. For the latter two columns, the selection is either based on the $N U V-V$ and/or the optical CMD, see the text for details. For the Xray and CV source numbers, we used the coordinates given in Cohn et al. (2010), but note that not all X-ray sources have a $F U V, N U V$ or optical counterpart.

\begin{tabular}{cccc}
\hline population & full $F U V$ & $5.5^{\prime \prime}$ & $19.8^{\prime \prime}$ \\
\hline MS & 155 & 83 & 588 \\
BS & 10 & 8 & 14 \\
HB & 1 & 0 & 6 \\
X-ray & 14 & 4 & 25 \\
CV & 6 & 3 & 7 \\
WDs & 16 & 5 & 19 \\
\hline
\end{tabular}

Table 3. Probabilities in \% returned by the KS test for the comparison of the various stellar populations located in the inner 5.5" circular area (col. 2) and the inner 19.8" (col. 3).

\begin{tabular}{ccc}
\hline population & $5.5^{\prime \prime}$ & $19.8^{\prime \prime}$ \\
\hline MS - BS & 10.3 & 0.2 \\
MS - WD & 91.4 & 8 \\
MS - CV & 50.0 & 0.9 \\
MS - HB & - & 40.1 \\
MS - X-ray & 34.7 & 30.1 \\
BS - WD & 36.6 & 12.2 \\
BS - CV & 38.5 & 49.2 \\
BS - HB & - & 0.4 \\
BS - X-ray & 74.0 & 1.9 \\
WD - CV & 94.7 & 39.9 \\
WD - HB & - & 6.3 \\
WD - X-ray & 99.4 & 68.3 \\
CV - HB & - & 0.8 \\
CV - X-ray & 96.1 & 11.1 \\
HB - X-ray & - & 22.7 \\
\hline
\end{tabular}

the probability that the maximum difference should be as large as observed under the hypothesis that both distributions are drawn from the same parent distribution. Thus, the higher the probability returned, the more similar are the two distributions. The smaller the probability, the less likely it is that the two distributions are drawn from the same parent distribution, i.e. the more different they are.

Interestingly, X-ray sources are less concentrated than WDs within the $19.8^{\prime \prime}$ circular area. The KS test returns a probability of $68 \%$ that both the WD and X-ray distributions are drawn from the same parent distribution. This is not statistically significant. However, for the inner 5.5", the KS probability is $99.4 \%$, closer to a $3 \sigma$ significance $(99.87 \%)$. If we use the MS sample as the reference population, then it appears that the X-ray sources are more centrally concentrated within the inner 5.5" (34.7\%) than the WDs $(91.4 \%$, more similar to the MS sample), and the CVs are in between $(50 \%)$.

The smallest probability was returned for the larger $19.8^{\prime \prime}$ area for the MS vs. BSs sample (0.2\%), BSs vs. HB stars (0.4\%), CVs vs. HB stars (0.8\%), and MS vs. CVs $(0.9 \%)$, indicating the difference in radial distribution (and hence in mass) between these populations.
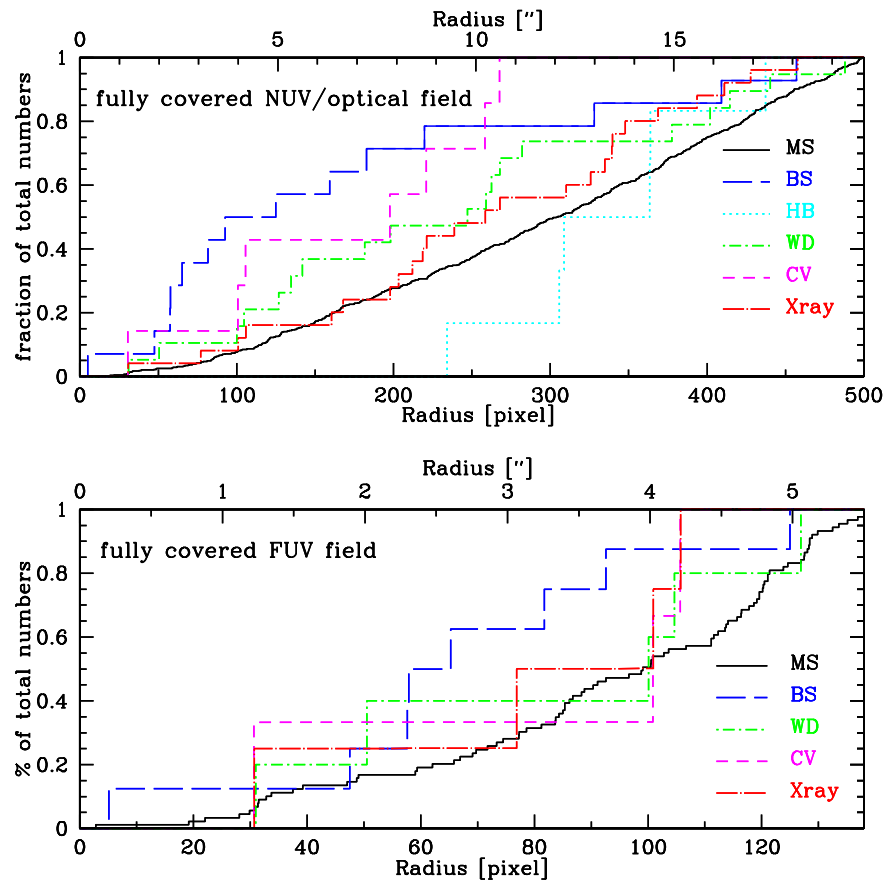

Figure 7. Top panel: Radial distribution of the various stellar populations within the inner $19.8^{\prime \prime}$ that is fully covered by the $N U V$ and optical data but not the $F U V$ data. Bottom panel: Radial distribution within the inner 5.5" circular area that is also fully covered by the $F U V$ master image.

\section{SUMMARY}

We have carried out a $F U V$ and $N U V$ photometric study on a field centered on the GC NGC 6397. 192 FUV sources were detected, of which 182 have a $N U V$ counterpart. The $F U V-N U V$ CMD, the first presented for this cluster, shows a sequence of MS stars and 16 WDs and gap sources. The three brightest $F U V$ sources are saturated in the $N U V$ so that we cannot clearly classify them as BSs or blue HB stars based on the FUV - NUV CMD alone. We matched our FUV $N U V$ catalogue to optical ACS data, which confirmed the classification of eight BSs. The optical CMD suggests that two of the three brightest $F U V$ sources are likely bright BSs, resulting in $10 \mathrm{BSs}$ detected in the $F U V$, but the brightest $F U V$ source is most likely a blue HB star.

Eighteen X-ray sources are located within our $F U V$ field of view, ten of those have a $F U V$ counterpart, and 13 have a $N U V$ counterpart, nine of those have both a $F U V$ and a NUV match. Five (U17, U19, U21, U22, and U23) of the six CVs in the FUV field of view were detected in both the $F U V$ and $N U V$. All of these five sources are located in the $\mathrm{WD} /$ gap area in our $F U V-N U V \mathrm{CMD}$, and in the gap between the WD and MS sequence in the $N U V-V$ CMDs. The sixth CV U25 has only a $N U V$ but no FUV counterpart. We clearly detect the $N U V$ counterpart to the likely MSP U18 (Cohn et al. 2010). A faint "smudge" can be seen in the FUV master image at the position of the MSP, but we did not assign a FUV magnitude. All CVs and the MSP show shorttimescale variability of the order of tens of a magnitude in their $N U V$ light-curves, i.e. typical flickering behaviour that is characteristic for CVs. Two of the CVs have previously 
been reported to show DN outbursts (Shara et al. 2005), however, none of the CVs exhibits any large-scale variability indicative of a DN outburst in our NUV light-curves.

The radial distributions suggest that the BSs and then the CVs are the most centrally concentrated population in both the inner $5.5^{\prime \prime}$ that are fully covered by the FUV data, and a larger 19.8" annulus that is covered by the $N U V$ and optical data. The HB stars, on the other hand, are the least concentrated population and in fact absent in the inner 5.5" annulus. The central concentration of BSs and CVs likely reflects their larger masses, compared to the smaller masses of the more widely distributed HB stars. The preferred location of the BSs and CVs towards the cluster centre might be an effect of mass segregation, i.e. more massive stars travel towards the cluster centre, and/or of their formation, as BSs and CVs likely form via dynamical processes in the dense cluster centre.

\section{ACKNOWLEDGEMENTS}

A.D. acknowledges support from the People Program (Marie Curie Actions) of the European Union's Seventh Framework Program FP7-PEOPLE-2013-IEF under REA grant agreement number 629579. We thank Jan Pflamm-Altenburg for helpful discussions.

\section{REFERENCES}

Anderson, J., Sarajedini, A., Bedin, L. R., King, I. R., Piotto, G. et al. 2008, AJ, 135, 2055

Bogdanov, S., van den Berg, M., Heinke, C. O., et al. 2010, ApJ, 709,241

Brown, T. M., Sweigart, A. V., Lanz, T., Landsman, W. B., \& Hubeny, I. 2001, ApJ, 562, 368

Cohn, H. N., Lugger, P. M., Couch, S. M., et al. 2010, ApJ, 722, 20

Cool, A. M., Grindlay, J. E., Cohn, H. N., Lugger, P. M. \& Slavin, S. D. 1995, ApJ, 439, 695

Davies, M. B. 1997, MNRAS, 288, 117

de Rijcke, S., Buyle, P. \& Dejonghe, H. 2006, MNRAS, 368, 43

Dieball, A., Knigge, C., Zurek, D. R., Shara, M. M., \& Long, K. S. 2005a, ApJ, 625, 156

Dieball, A., Knigge, C., Zurek, D. R., et al. 2005b, ApJ, 634, L105

Dieball, A., Knigge, C., Zurek, D. R., et al. 2007, ApJ, 670, 379

Dieball, A., Long, K. S., Knigge, C., Thomson, G. S., \& Zurek, D. R. 2010, ApJ, 710, 332

Di Stefano, R., \& Rappaport, S. 1994, ApJ, 423, 274

Dolphin, A. E. 2000, PASP, 112, 1383

Dorman, B. 1992, ApJS, 81, 221

Edmonds, P. D., Gilliland, R. L., Heinke, C. O., \& Grindlay, J. E. 2003a, ApJ, 596, 1177

Edmonds, P. D., Gilliland, R. L., Heinke, C. O., \& Grindlay, J. E. 2003b, ApJ, 596, 1197

Elsner, R. F., Heinke, C. O., Cohn, H. N., et al. 2008, ApJ, 687, 1019

Elson, R., Hut, P., \& Inagaki, S. 1987, ARA\&A, 25, 565

Ferraro, F. R., Sills, A., Rood, R., Paltrinieri, B., \& Buonanno, R. 2003, ApJ, 588, 464

Forestell, L. M., Heinke, C. O., Cohn, H. N., et al. 2014, MNRAS, 441, 757

Gänsicke, B. T., Beuermann, K., \& de Martino, D. 1995, A\&A, 303,127

Gehrels N. 1986, ApJ, 303, 336

Giersz, M. \& Heggie, D. 2009, MNRAS, 395, 1173
Gosnell, N. M., Mathieu, R. D., Geller, A. M., Sills, A., Leigh, N. \& Knigge, C. 2015, ApJ, 814, 163

Gratton, R. G., Bragaglia, A., Carretta, E., Clementini, G., Desidera, S., Grundahl, F. \& Lucatello, S. 2003, A\&A, 408, 529

Grindlay, J. E., Heinke, C., Edmonds, P. D., \& Murray, S. S. 2001a, Science, 292, 2290

Grindlay, J. E., Heinke, C., Edmonds, P. D., Murray, S. S., \& Cool, A. M. 2001b, ApJ, 563, L53

Grindlay, J. E. 2006, Advances in Space Research, 38, 2923

Hannikainen, D. C., Charles, P. A., van Zyl, L., et al. 2005, MNRAS, 357, 325

Hansen, B. M. S., Anderson, J., Dotter, A. et al. 2007, ApJ, 671, 380

Hansen, B. M. S., Kalirai, J. S., Anderson, J., et al. 2013, Nature, 500,51

Harris, W. E. 1996, AJ, 112, 1487

Heggie, D. \& Giersz, M. 2009, MNRAS, 397, 46

Heinke, C. O., Edmonds, P. D., Grindlay, J. E., et al. 2003a, ApJ, 590,809

Heinke, C. O., Grindlay, J. E., Edmonds, P. D., et al. 2003b, ApJ, 598,516

Heyl, J. S., Richer, H., Anderson, J., et al. 2012, ApJ, 761, 51

Hills, J. G., \& Day, C. A. 1976, Astrophys. Lett., 17, 87

Hurley, J. R., Shara, M. M., Richer, H. B. et al. 2008, AJ, 135, 2129

Husser, T.-O., Kamann, S., Dreizler, S. et al. 2016, A\&A, 588, 148

Hut, P., McMillan, S., Goodman, J., et al. 1992, PASP, 104, 981

Ivanova, N., Heinke, C. O., Rasio, F. A., et al. 2006, MNRAS, 372,1043

Kalirai, J. S., Anderson, J., Richer, H. B. et al. 2007, ApJ, 657, 93

Kamann, S., Husser, T.-O., Brinchmann, J., et al. 2016, A\&A, 588, A149

Knigge, C., Zurek, D. R., Shara, M. M., \& Long, K. S. 2002, ApJ, 579,752

Knigge, C., Dieball, A., Maíz Apellániz, J., Long, K. S., Zurek, D. R. \& Shara, M. M. 2008, ApJ, 683, 1006

Lind, K., Charbonnel, C., Decressin, T., Primas, F., Grundahl, F. \& Asplund, M. 2011, A\&A, 527, 148

Lugger, P. M., Cohn, H. N., Heinke, C. O., Grindlay, J. E., \& Edmonds, P. D. 2007, ApJ, 657, 286

Maccarone, T. J., Long, K. S., Knigge, C., Dieball, A., \& Zurek, D. R. 2010, MNRAS, 406, 2087

McCrea, W. H. 1964, MNRAS, 128, 147

Milone, A. P., Villanova, S., Bedin, L. R., Piotto, G., Carraro, G., Anderson, J., King, I. R. \& Zaggia, S. 2006, A\&A, 456, 517

Milone, A. P., Marino, A. F., Piotto, G., et al. 2012, ApJ, 745, 27

Pooley, D., Lewin, W. H. G., Verbunt, F., et al. 2002, ApJ, 573, 184

Pooley, D., \& Hut, P. 2006, ApJ, 646, L143

Richer, H, Dotter, A., Hurley, J. et al. 2008, ApJ, 135, 2141

Richer, H. B., Goldsbury, R., Heyl, J., et al. 2013, ApJ, 778, 104

Sarajedini, A., Bedin, L. R., Chaboyer, B., Dotter, A., Siegel, M. et al. 2007, AJ, 133, 1658

Servillat, M., Dieball, A., Webb, N. A., et al. 2008, A\&A, 490, 641

Shara, M. M., Hinkley, S., Zurek, D. R., Knigge, C., \& Dieball, A. 2005, AJ, 130, 1829

Shara, M. M., \& Hurley, J. R. 2006, ApJ, 646, 464

Stetson, P. B. 1987, BAAS, 19, 745

Strickler, R. R., Cool, A. M., Anderson, J., Cohn, H. N., Lugger, P. M. \& Sterenelli, A. M. 2009, ApJ, 699, 40

Thomson, G. S., Dieball, A., Knigge, C., Long, K. S., \& Zurek, D. R. 2010, MNRAS, 406, 1084

Thomson, G. S., Knigge, C., Dieball, A., et al. 2012, MNRAS, 423, 2901 
Torres, S., García-Berro, E., Althaus, L. G., \& Camisassa, M. E. 2015, A\&A, 581, A90

Tout, C. A., Pols, O. R., Eggleton, P. P., \& Han, Z. 1996, MNRAS, 281,257

Warner, B. 2003, Cataclysmic Variable Stars

Winget, D. E.,Kepler, S. O., Campos, F., Montgomery, M. H., Girardi, L., Bergeron, P., \& Williams K. 2009, ApJ, 693, 6

Wood, M. A. 1995, White Dwarfs, 443, 41

Zhang, P. F., Xin, Y. L., Fu, L., et al. 2016, MNRAS, 459, 99

Zurek, D. R., Knigge, C., Maccarone, T. J., Dieball, A., \& Long, K. S. 2009, ApJ, 699, 1113

Zurek, D. R., Knigge, C., Maccarone, T. J., et al. 2016, MNRAS, 460,3660

This paper has been typeset from a $\mathrm{T}_{\mathrm{E}} \mathrm{X} / \mathrm{LAT} \mathrm{T} \mathrm{X}$ file prepared by the author. 